\title{
Pierre Bourdieu y el análisis de los campos intelectuales*
}

\author{
Homo academicus and the analysis \\ of intellectual fields \\ José Luis Moreno Pestaña \\ (Universidad de Cádiz)
}

Recibido: 24/01/2011

Aceptado: 07/11/2012

\section{Resumen}

Homo academicus propone una reflexión epistemológica de interés para investigaciones similares y para la práctica sociológica en general. En segundo lugar, propone un mapa del mundo universitario francés, de las diferentes formas de capital que lo configuran y de los diversos tipos de carrera académica. Más allá del origen nacional de su análisis, Bourdieu insiste en el valor del modelo. En tercer lugar, Bourdieu ofrece una explicación de un acontecimiento histórico que trasciende la universidad, pero en el que ésta jugó un papel de primer orden. Mayo del 68 muestra cómo un acontecimiento histórico desestabiliza parcialmente el orden social existente. Este artículo interroga Homo academicus desde un punto de vista epistemológico, estructural e histórico.

Palabras clave: sociología del campo intelectual, Pierre Bourdieu, epistemología de las ciencias sociales.

\begin{abstract}
Homo academicus proposed an epistemological reflection that is of interest to both further research on the object itself as well as the practice of sociology in gen-

\footnotetext{
* Texto escrito en el marco del proyecto de I+D FFI2010-15196. Una versión francesa de este texto titulada "Ordre épistémologique, ordre structural et ordre historique dans Homo academicus" apareció en Frédéric Lebaron y Gérard Mauger, Lectures de Bourdieu, París, Ellipses, 2012, pp. 263-278 y una inglesa en la siguiente referencia: "Homo academicus and the analysis of intellectual fields", Academia, 2 (1), pp. 69-92.
} 
eral. Second, he proposed a map of the university world in France, the various forms of capital that organise it and the most customary types of career paths. Despite the national basis of his analysis, Bourdieu insisted that it could be extended beyond France. Third, he offered an explanation of a historical event that spilled over from the university campus, yet one in which the university played a major role; the uprising of May " 68 showed how a historic event that partially destabilised the social order occurred. Epistemological, structural and historical orders - these are the angles from which Homo academicus shall be read.

Keywords: sociology of intellectual field, Pierre Bourdieu, epistemology of social sciences.

Homo academicus fue un libro difícil de escribir para Pierre Bourdieu. Lo escribió él, pero no lo trabajó solo. La investigación fue realizada con Yvette Delsault (2002: 229-230), en la época una colaboradora muy cercana. Bourdieu ofreció a Delsault aparecer como coautora del libro, a lo que ésta se negó, por desacuerdo con el tono final del libro, demasiado "violento" y "masculino" para asociarle su nombre. Pese a ello, Delsault reconoce que se trata, como Bourdieu insiste en la entrevista entre ambos, de un libro elaborado con enormes precauciones metodológicas. He aquí, pues, una obra bifronte, por un lado, humana demasiado humana, por otro lado, científicamente muy controlada. No se analizará aquí la primera dimensión, que requeriría una sociología de la sociología del mundo académico propuesta por Bourdieu: de si ésta debe mucho o no a la posición y a la trayectoria de su autor, de si los instrumentos metodológicos y los conceptos utilizados por Bourdieu son los más adecuados para acometer dicha empresa. Trataremos la segunda dimensión, es decir, las aportaciones científicas de Bourdieu al conocimiento del mundo académico e intelectual.

Las contribuciones de Bourdieu son de tres órdenes. En primer lugar, Bourdieu propone una reflexión epistemológica interesante tanto para otras investigaciones sobre el mismo objeto como para la práctica de la sociología en general. En segundo lugar, Bourdieu propone un mapa del mundo universitario francés, de las diversas formas de capital que lo organizan y de los tipos de trayectoria más habituales. Bourdieu insiste en que pese a que su análisis es nacional, resulta posible extenderlo más allá del Hexágono. En tercer lugar, Bourdieu propone una explicación de un acontecimiento histórico que desborda el campo universitario, pero en el que éste juega un papel muy relevante. Con el estallido de Mayo del 68, puede verse cómo se produce un acontecimiento histórico en el cual, parcialmente, se desestabiliza el orden social. Orden epistemológico, orden estructural y orden histórico, tales son los ángulos desde los que se leerá Homo academicus. 


\section{Convertir los individuos empíricos en epistémicos}

Analizar sociológicamente la realidad, explica Bourdieu, supone reformularla según ciertos parámetros que nos resultan relevantes. Ese trabajo permite clasificar a todos los individuos según idénticos criterios. Por supuesto, existen múltiples maneras de clasificar a los individuos en un marco científico de análisis. Bourdieu asume como evidente la pluralidad científica en el proceso de construcción de nombres en sociología. Esos nombres no son como los nombres de uso cotidiano: no designan individuos empíricos con sus personalidades complejas y difíciles de cernir en un conjunto de predicados. Un nombre científico designa un conjunto de propiedades que producen efectos en un campo. Bautiza así a a los agentes como individuos epistémicos. La diferencia entre los individuos empíricos y los epistémicos ayuda a comprender en qué los conceptos científicos se distinguen de los epítetos del lenguaje común. Dos individuos empíricos se encuentran separados por nombres propios que no dan información de sus diferencias. Un concepto científico agrupa individuos empíricos diversos que comparten un conjunto de rasgos que, desde ese punto de vista, los hace equivalentes, en tanto que agentes dentro de un plano de la realidad. El concepto científico rebautiza al individuo empírico y lo convierte en un agente eficiente en un espacio social, es decir, en un individuo epistémico

El mundo social en general y el mundo universitario en particular, se encuentran repletos de clasificaciones, empezando por los puestos universitarios. Estos consagran a los individuos como catedráticos o como profesores, sin embargo esos nombres comunes ocultan individuos con propiedades diferentes: uno puede tener una influencia científica real mientras otro es un gestor universitario y aquel dedica su tiempo a la intervención periodística. En realidad, bajo la común apelación se encuentran propiedades muy distintas e intereses por mundos sociales divergentes. Se "llaman" igual pero tienen competencias diferentes y se vuelcan en realidades distintas (HA, 63). Esos nombres no son triviales sino que sostienen jerarquías sociales. Por ejemplo, se supone que el profesor sabe más que el ayudante, o que el investigador se orienta por exigencias científicas: quizá lo hace según las exigencias de la política universitaria local e invierte más tiempos en contactos sociales que en leer y en publicar. Así, el nombre científico permite ver la realidad de otro modo a como lo permiten las clasificaciones corrientes. Clasificaciones que, por lo demás, se encuentran en conflicto, pues cada uno de los agentes pretende imponer aquellas que más convienen a sus propios intereses. En el caso del mundo universitario, Bourdieu indica que la falta de referencias compartidas -y la multiplicación cacofónica de parámetros de juicio distintos- para jerarquizar los méritos oficia como un sistema de defensa compartido, que permite a los sujetos obviar su verdadera posición recurriendo al sistema de clasificación que mejor le permite salvar la cara ante sí mismo y ante los demás (HA, 104). 
¿Significa esto que los individuos epistémicos permiten ver la realidad social mientras que en la vida cotidiana quedaría embrollada? La respuesta, para Bourdieu, es sí y no. La respuesta positiva la dan los propios analizados que, en ocasiones, reconocen en las explicaciones del sociólogo planos de su existencia. Estos les pasan desapercibidos normalmente, ya que los individuos se orientan sin demasiada distancia reflexiva con las urgencias cotidianas (HA, 38). Si se quedara en este momento de su argumentación, Bourdieu consideraría que su construcción científica mostraría la verdad del mundo, con la única reserva de que no podemos confundir lo descubierto por el análisis con la intención consciente de los agentes.

Entonces, ¿dónde queda la conciencia de que la perspectiva sociológica es una construcción que, como tal, no pretende ser un espejo de la naturaleza del mundo social? En dos planos: por una parte, los individuos epistémicos convierten en discretas propiedades continuas y separan rasgos que en la realidad no se encuentran distribuidos de manera tan precisa. Es decir, los individuos epistémicos no se superponen completamente con los individuos empíricos, como si estos fueran el ejemplar repetible e intercambiable de la especie recogida en el concepto sociológico. Bourdieu (HA, 40-41) no lo señala pero se encuentra muy cerca de una de las ideas centrales de la epistemología de Jean-Claude Passeron (2006: 89-123). Los nombres de la sociología son mixtos lógicos, que por una parte designan como los nombres comunes un conjunto de características generales (por ejemplo, agentes con estructura de capital dominada por el poder académico), pero, por otra parte, sólo tienen valor, si no quieren convertirse en conceptos empíricamente infecundos, cuando los referimos a un conjunto de operaciones de investigación localizadas en el espacio y en el tiempo (y, en ese sentido, funcionan como un nombre propio: estos conceptos para analizar a estos sujetos, tras haberlos analizado según estas pautas). En suma, los nombres que impone el sociólogo -en su rebautizo epistémico de los individuos empíricos- no son los únicos legítimos.

Además, el sociólogo, máxime cuando estudia su propio universo, debe precaverse, por la vía de explicitarlos, ante tres tipos de sesgos que siempre acompañan su investigación. Ese trabajo de clarificación es inagotable y, por tanto, nunca se accede al punto donde es posible contemplar todas las perspectivas parciales sobre el mundo sin quedarse atrapado por alguna. En primer lugar, debido a sesgos derivados de su propia trayectoria social y científica, algo que Bourdieu $(2001,2004)$ pretendió terminar de analizar en el último de sus libros publicados así como en su curso sobre la ciencia. En segundo lugar, a causa de los supuestos implícitos en los conceptos empleados: por ejemplo, alguien que confunda la determinación social con la ausencia de lucidez (el agente como simple portador de estructuras) no puede comprender el concepto de habitus, que parte de la tesis de que tenemos grados variables de comprensión de nuestras determinaciones sociales y medios de cálculo parciales para seguir, contrarrestar u oponernos a estas. Por tanto, un supuesto 
(una idea filosófica de la libertad: libertad como no condicionamiento absoluto) se encuentra detrás de muchas críticas de quienes no comprenden los supuestos filosóficos de la noción de habitus y creen que éste funciona como un cepo tiránico que mata toda creatividad y toda lucidez en los sujetos. En tercer lugar, el investigador debe renunciar a utilizar la ciencia para influir en el mundo científico y para promover sus propios intereses. Es el caso, explica Bourdieu (HA, 29-30), de un trabajo de Raymond Boudon en el que éste denuncia las celebridades francesas y adula las internacionales (fundamentalmente norteamericanas), sin mostrar lo que le va en el envite (él se vería revalorizado si triunfase su visión de las buenos y los malos ranking) y sin pararse un segundo a examinar empíricamente si la realidad resiste a sus análisis (algo que sucede, según Bourdieu, ya que, por ejemplo, los investigadores menos valorados por los científicos franceses son los que más impacto tienen en el muy internacional Citation Index).

\section{Distancias y proximidades en el campo del poder académico}

\subsection{Facultades en conflicto}

Dos especies de capital (el económico y el cultural) organizan el campo del poder. Los profesores universitarios se encuentran más próximos de la segunda, por ende, en el polo dominado del campo del poder. Este se encuentra también internamente dividido entre los sectores más dependientes del mercado cultural (por ejemplo, escritores y periodistas) y aquellos que, como los universitarios, tienen un respaldo institucional. Las distancias entre ambos sectores dependen de coyunturas históricas. La separación fue máxima al final del siglo XIX -periodo en el que creció la distancia entre el poder académico y el político- hasta el periodo de entreguerras en el siglo XX. Posteriormente, el crecimiento de la población estudiantil obligó al reclutamiento de profesores menos académicos, procedentes del medio cultural y periodístico. De hecho, cada época, insiste Bourdieu, podría estudiarse según las relaciones existentes entre el mundo académico y el cultural, cuyos indicadores podrían estar en la comparación del origen social y escolar de los pobladores de ambos territorios, de los pasos entre un campo y otro, de los lugares de encuentro, de la concepción que ambas poblaciones se hacen de su éxito (según un estudio citado de Alain Girard, los escritores suelen tener una visión más carismática, los profesores lo achacan a sus maestros y sus familias), etc.

La división entre lo económico y lo cultural, que estructura el campo del poder, se reproduce entre las facultades universitarias y dentro de cada facultad. Es decir, Bourdieu, por momentos, parece razonar como si cada realidad tuviera dentro de sí los mismos principios de diferenciación con los que ha construido su objeto el 
sociólogo... y nada más. Bourdieu fue un gran admirador de Gottfried W. Leibniz quien afirmaba en la Monadologie ( $\$ 56$ ) que cada sustancia expresa en su interior todo el universo y que mirando dentro de ella podríamos contemplar un espejo del conjunto de la realidad. Por eso, si tuviéramos una perspectiva infinita y conociéramos todas las circunstancias en las que se encuentra un ser concreto, predeciríamos la dirección de su trayectoria futura. De ser así, la realidad perdería su carácter histórico y se convertiría en la reiteración monótona de idénticos principios de diferenciación, fuera cual fuera el tiempo, el espacio o el lugar; cuando tales diferenciaciones no se produzcan se debería a que nuestro conocimiento de la realidad no era completo. Indudablemente, Bourdieu puede defender que sólo desarrolla lógicamente los principios que ha elegido para construir su objeto de estudio, aunque otras perspectivas serían posibles. Como ya hemos señalado, esa posición sería coherente con la lectura realizada aquí de su introducción epistemológica ${ }^{1}$.

Volvamos al análisis de Bourdieu. El campo universitario reproduce el campo del poder y así las facultades de ciencias están dominadas desde el punto de vista temporal (político y económico) por las de Medicina o Derecho, esto es, las más cercanas del campo del poder económico tout court; cuando la jerarquía es científica las relaciones se invierten. Las facultades de Letras quedan a mitad de camino entre ambas.

Bourdieu muestra cómo cada posición recluta los tipos humanos que requiere para mantener su estabilidad. Las instituciones son modos de generación de habitus similares. La Facultad de Medicina transmite conocimientos, pero también un modo de vida acorde con los valores dominantes, algo que muestra la preponderancia católica dentro del cuerpo. Los profesores de Derecho, surgidos en su gran mayoría de la burguesía, tienden a acumular puestos universitarios, políticos e incluso en los negocios. Los profesores de ciencias y letras que proceden, ya sea de las clases medias o populares, ya sea del propio medio universitario, tienden, por el contrario, a consagrarse a la institución de la que procede su movilidad social ascendente. En fin, los que más invierten en la vida científica tienden al celibato, mientras que la familia extensa acompaña como signo de su integración social a los universitarios que se proyectan en la política o en el mundo del capital.

Cada Facultad, por lo demás, define lo que son las actividades de investigación. Este es uno de los puntos donde se muestra la fecundidad empírica del modelo analítico de Bourdieu. La coexistencia de definiciones distintas de los que es investi-

\footnotetext{
${ }^{1}$ En cualquier caso, el lector tiene derecho a dudar del recurso constante a las homologías (toda realidad tiene divisiones internas que se superponen con las de otras) y de preguntarse si el trabajo sociológico no sirve de ejemplo a una cierta metafísica. En ese caso, las críticas que sin nombrarlo le dirige Jean-Claude Passeron (2006: 310-311) estarían completamente justificadas. Véase una consideración crítica y equilibrada de la utilización del Análisis de Correspondencias Múltiples en Homo academicus en Baranger (2009: 71-72).
} 
gar, es un tema clásico de la sociología del conocimiento. Como explicaba Randall Collins (2000: 523-569, 874-877), la investigación en las ciencias con un solo paradigma permite la concentración colectiva en un problema (son las "ciencias del descubrimiento rápido"). La investigación acompaña las innovaciones tecnológicas, que se convierten en el criterio del buen hacer científico. La notación matemática permitió la creación de un lenguaje común y resolvió en cierta medida la cuestión, siempre delicada, de cómo traducir lingüísticamente los resultados empíricos. El trabajo en equipos se volvió posible y la concentración tecnológica de la actividad permitió una relativa indiferencia frente a las cuestiones teóricas. A veces existe una alta conciencia teórica del trabajo de los equipos científicos, mientras que en ocasiones el trabajo intelectual se reduce a elaborar retrospectivamente los resultados obtenidos con las tecnologías.

Bourdieu considera que ese modelo, procedente de las ciencias de la naturaleza, ha invadido el conjunto de la actividad científica, homogeneizando realidades muy disímiles, que se redefinen en cada una de las facultades. Muchos investigadores no son sino patrones que pasan su vida persiguiendo financiación $-\mathrm{y}$ cultivando el capital social- para que otros investiguen. Así, la vida científica se encuentra más cercana de la gestión de una clientela y de la conexión con redes políticas y económicas que permiten mantenerla. La lógica del capital social predomina sobre los problemas de investigación. Tal es el perfil del patrón tolerante, heredero de redes de capital social (familiares, universitarias), que encubre su falta de compromiso efectivo con la ciencia con profesiones de antidogmatismo teórico y político (definirse firmemente acerca de algo conflictivo, debe evitarse cuando se cultiva el capital social) y fundamentalmente preocupado por la calidad de sus contactos (ya sean los estudiantes-clientes, ya sean los centros donde lograr los recursos). La formación científica e intelectual de los estudiantes resultan menos importantes que la promesa de gestión de una carrera. Por tanto, para poder entrar en dichas redes de reclutamiento, se necesita mostrar que se comparten los valores del grupo: el origen social -formar parte de buena familia- es un criterio, la docilidad entusiasta respecto de la integración grupal es otro (HA, 79). La cooptación, fundamentalmente en Medicina o Derecho, no promueve competencias científicas sino modos de ser globales, hábitos. Dichas facultades, que reclutan por currículos implícitos, son más selectivas socialmente que aquellas donde las competencias requeridas, caso de las ciencias, se encuentran más formalizadas y no se trasmiten por medio de la acción pedagógica familiar. ${ }^{2}$ Las distintas facultades y dentro de éstas de los diferentes polos (más espirituales o temporales) se instalan en el dilema de, o bien producir

\footnotetext{
2 La pasión de Bourdieu por la sociología, enemiga del ensayismo inspirado, deriva de una creencia en la racionalidad científica compartida pero también un compromiso social igualitario con aquellos que sólo disponían de su esfuerzo escolar para producir discursos sobre la realidad. Véase Bourdieu (1980: 11).
} 
agentes socialmente integrados para ejecutar las funciones establecidas, o bien guiarse por la lógica de la racionalidad científica. La responsabilidad social y la responsabilidad científica se enfrentan en un juego de suma cero. Cuanto más alta es una, más baja es otra. No hace falta insistir en lo lejos que Bourdieu se encuentra de las críticas radicales al poder de la ciencia que procedentes de fuentes ideológicas dispares (que van desde la extrema derecha a la extrema izquierda), se amplificaron en Mayo del 68 y configuraron la nebulosa posmoderna, por la que Bourdieu tuvo siempre una enorme hostilidad.

\subsection{Los poderes y las letras}

Como ejemplo del conflicto entre los dos tipos de poderes, Bourdieu se concentra en las facultades de Letras de 1967. En éstas, supone Bourdieu, su modelo se mostraría en toda su pureza, siendo mucho más difícil encontrarlo en otras facultades (HA, 106). En Letras existe una división más marcada entre aquellos centrados en el trabajo científico y cultural y los que controlan la política universitaria (como la gestión, la incorporación de docentes y becarios, etc.). Bourdieu acota su universo de acuerdo con su visión teórica. Dado que la universidad francesa está jerarquizada y centralizada, la mayoría de la población retenida procede de París, de las grandes instituciones universitarias y de los profesores más prestigiosos. Así Bourdieu pretende reproducir con su universo la estructura de la realidad estudiada, huyendo de la selección por bola de nieve (que termina premiando las formas más mundanas de prestigio) o al azar. Ésta última daría idéntica representación a individuos con poderes muy diferentes y de ese modo haría equivalentes propiedades que no se encuentran "valoradas" del mismo modo por el campo académico. Por lo demás, Bourdieu insiste en que las relaciones de poder no se comprenden por medio de los individuos, sino analizando el conjunto de propiedades eficientes en el campo y su distribución diferencial.

Con la población estudiada, Bourdieu no explica cómo en dicho universo tan jerarquizado puede subsistirse en los escalafones inferiores. El supuesto básico no es otro que la existencia de una sociedad académica donde los de abajo y los de arriba persiguen lo mismo, y según idénticos criterios. Aquellos que no lo logran, es decir, que no dejan su rastro en las jerarquías académicas dominantes, no tienen información alguna que ofrecerle a Bourdieu, salvo testimonio de su miseria o, lo que viene a ser lo mismo, discursos de racionalización. Esos discursos de racionalización impiden la existencia de un sistema de evaluación compartido, que obligaría a todos a conocer su verdadera posición. Pero quizá podría pensarse que Bourdieu no contempla las formas plurales de vivir en la universidad.

La opción metodológica de Bourdieu es razonable en lo que concierne al poder institucional: el Rector de la Universidad de París tiene mayor influencia que un jefe 
de Departamento y es absurdo darle a las propiedades de ambos el mismo peso en una investigación. Si se trata de la notoriedad intelectual, tampoco cabe discutirle que se concentre en quienes traspasan un cierto umbral (por ejemplo, más de 5 citas en el Citation Index). ${ }^{3}$ Otra cuestión es si el prestigio científico podría, como él cree, medirse de ese modo. ¿Tener el reconocimiento de los pares -que es lo que recoge el Citation Index - durante un cierto tiempo equivale a un trabajo intelectual productivo? Bourdieu se encuentra, en este punto, muy cerca de Randall Collins (2000: 85-87), quien considera que sólo un conjunto de individuos (aquellos que acceden al centro del espacio de atención), representantes de una posición (las escuelas en conflicto están entre tres y seis, de lo contrario no hay posibilidad de puntos comunes de discusión), copan la atención intelectual en un período. Del resto puede prescindirse, aunque Collins reconoce que alguna de las estrellas intelectuales eran poco reconocidas en su tiempo (Moreno Pestaña, 2009). Se piense lo que se piense de si las redes de productividad intelectual se confunden con las redes de notoriedad en los contemporáneos -algo más que dudoso ${ }^{4}$, cierto es que Bourdieu proporciona un mapa muy reducido de las pasiones académicas e intelectuales y que otra selección de la población -y otro marco teórico- nos darían una visión muy diferente de la vida universitaria y de la libido institucional e intelectual de sus agentes.

¿Cómo funciona el poder puramente universitario, según el modelo de Bourdieu? Por medio de la ocupación de posiciones que permiten determinar el acceso de otros sujetos a determinadas posiciones. Los individuos más dotados de capital universitario, a menudo poco estimados como científicos, establecen sus clientelas intercambiando servicios entre sí. A menudo esas relaciones comenzaron en la ENS, centro de socialización de la elite universitaria francesa que no existe en otros países. La edad diferencia a los detentadores del poder académico, ya que el acceso a posiciones de poder requiere tiempo y respetar el orden lógico de la sucesión, sin pretender pasarse en rapidez. La competencia, insiste Bourdieu, es la condición del orden, ya que quienes compiten admiten un valor compartido y, además, son los dominantes quienes regulan la competición.

¿Cómo regulan los dominantes la competencia universitaria? Jugando con las esperanzas y con los tiempos de acceso a los puestos. Para ello, las posibilidades tienen que ser restringidas, de modo que los agentes puedan formarse expectativas cir-

\footnotetext{
3 A partir de la población de catedráticos de universidad de París, Bourdieu (HA, 101) retiene a quienes tienen al menos dos de las propiedades que se detallan a continuación. En lo que se refiere al poder universitario: miembros del Institut de France, del tribuna de agregación, del tribunal de la École Normale Supérieure, del Comité consultivo de Universidades. En lo que respecta al poder científico: miembros de la comisión del CNRS entre 1963 y 1967. Y en lo que toca al poder científico: más de cinco apariciones en el Citation Index.

${ }^{4}$ Bourdieu es consciente del problema como lo muestra la nota 8 de la página 104 de Homo academicus, aunque decide no prestarle mucha atención.
} 
cunscritas y razonables (una posibilidad escasa no atrae a casi nadie) y, condición importantísima, relativamente indeterminadas, para que el patrón pueda manipular a los contendientes (a más contendientes, más poder del patrón). Los aspirantes quedan en situación de subordinación e infantilización ante un poder sin reglas definidas y que, por tanto, coloca a los sujetos en una situación de zozobra permanente, además de incitarlos a someterse aún más al patrón para desbancar a los contendientes.

Un patrón universitario - los hay que forman científicamente a sus discípulos y les ayudan a publicar, pero son los menos- debe moverse entre dos excesos, en los cuales, las fuentes de su poder pueden secarse. Por un lado, debe evitar obstaculizar demasiado la independencia de sus pupilos, si no quiere perder poder de atracción, pero, por otro lado, tampoco puede lanzarlos demasiado pronto a la vida universitaria adulta, si no quiere, uno, que el trabajo de infantilización no produzca su efecto de inculcación y dos, y derivado de lo anterior, tener un competidor autónomo disputándole al clientela. Los patrones universitarios, por malos entrenadores científicos que sean, atraen a los alumnos más avispados -que suelen ser también los más próximos socialmente-. El círculo de reproducción se cierra: los profesores tienden a reclutarse entre aquellos que comprenden el juego, eligen los temas de tesis y los patrones adecuados y, durante la carrera, saben esperar (asumiendo la dependencia y hasta las humillaciones de la infantilización, con sus consiguientes restricciones en otras áreas de la vida), pueden esperar (porque disponen de los medios económicos, familiares e institucionales para ello) y saben darle al tutor de su carrera las pruebas intelectuales, pero sobre todo sociales, de que serán discípulos dignos.

Tal es el habitus universitario, en la región más próxima del poder, y con él viene el conservadurismo intelectual, enemigo de todo cuanto se aparte de la "seriedad" académica, esto es, de los consensos prácticos que permiten los equilibrios académicos e intelectuales de los diversos polos de poder. Las redes institucionales, por lo demás, exigen que se invierta tiempo en ellas, dirigiendo tesis, asistiendo a conferencias y a tribunales, participando en revistas, y en todo ello y con todo ello, invitando a los colegas y aceptando sus invitaciones. Ese tiempo permite el reconocimiento institucional del grupo pero, obviamente, impide atender a la producción intelectual, condición del reconocimiento científico. El poder académico es, así, un poder temporal que ocupa un espacio que, en principio, estaba pensando para la producción intelectual. Consciente de su impostura, no deja de percibirse frágil y de ahí su ocasional agresividad frente a los creadores científicos.

Bourdieu explica que la adhesión ciega a la institución académica proviene, en buena medida, de las propiedades sociales de sus pobladores. Reclutados entre la pequeña burguesía, sin más capital cultural que el que proporciona la institución, satisfechos de su estado, la producción favorita de tales productores son los manuales de síntesis que, por un lado, sirven para reforzar las redes de capital social 
-mediante el reconocimiento de las "aportaciones" de los colegas- y, por otro lado, permiten dar salida a la producción necesaria para superar concursos y oposiciones. Se trata pues de una producción intelectual que permite ascender en la jerarquía de enseñantes y a la vez producirlos, sin olvidar los pingües beneficios económicos que genera.

La región universitaria más próxima del polo científico tiende a situarse -recuerdo: en la época y el lugar que estudia Bourdieu- en las disciplinas nuevas (sociología, etnología) o marginales, en suma, en aquellas donde los controles de acceso y las prácticas de inculcación de las rutinas son menores. Sus lugares institucionales se encuentran en el Collège de France y la École de Hautes Études en Sciences Sociales. La escasa carga docente permite la exploración científica y el establecimiento de un programa propio de trabajo acorde con los debates científicos dominantes. Las disputas por el espacio de atención, que diría Randall Collins, motivan a los agentes, dentro de un entorno que permite escasísimas consagraciones. Bourdieu considera que ese juego arriesgado solo puede afrontarlo una población que, confrontada con la de los profesores, aparece como socialmente más favorecida (HA, 143). Se confirma así una "ley" (que Bourdieu tiene en gran estima teórica) que restringe la propensión a los riesgos a la disposición de mayores capitales, ya que los creadores intelectuales y científicos no reciben demasiada recompensa institucional (Bourdieu recuerda la debilidad universitaria en aquel momento de Althusser, Foucault o Barthes).

¿La descripción de Bourdieu tiene un valor sociológico general o se restringe a la época que estudia? Como programa de trabajo tiene un gran valor. Saltando de época y lugar, la sociología histórica de las escuelas helenísticas realizada por Michel Foucault (2001: 111-112) ofrece una pista interesante para relacionar el origen social con el modo de practicar la filosofía y/o la religión. Las escuelas helenísticas generalizaron las prácticas de reflexión sobre la propia vida que el mundo ateniense reservaba a una cierta elite. Evidentemente, existía un umbral económico y cultural por debajo del cual era sumamente improbable -que no imposible- enrolarse en la vida intelectual. Una vez transpuesto dicho umbral se distinguían dos polos: uno, a menudo ocupado por personas de origen humilde, centrado en prácticas rituales de tipo religioso, en las que la adscripción sectaria y la celebración del culto primaban sobre el trabajo de transformación individual y sobre la producción filosófica, otro, en el que abundaban las clases pudientes, de mayor distancia respecto de las prácticas rituales, y más creativo en la modificación subjetiva y en la creación cultural. El proceso descrito por Foucault, diría Bourdieu, tiene un valor más general: las clases dominadas adquieren más seguridad en los entornos culturales mejor codificados y más definidos; las clases dominantes, debido a un capital cultural y a un sentido de la orientación precozmente adquirido, pueden permitirse una relación más distendida con la cultura. Sin embargo, como insiste Foucault y 
mostraría cualquier análisis histórico medianamente complejo, será un error convertir cierta tendencia en un tipo ideal dogmático, que presumiría tras lo dogmático y religioso la rigidez de las clases medias bajas y tras lo filosófico y creativo, las capacidades complejas de las clases cultural y económicamente privilegiadas. Los epicúreos, en su origen, tenían una composición popular, se distanciaban ostentosamente de la religión y produjeron una filosofía sofisticada. Por lo demás, la reflexión filosófica de las escuelas helenísticas, a menudo, estuvo unida a prácticas rituales y religiosas muy intensas. Del mismo modo, las organizaciones totales (conventos, internados, ghettos académicos, partidos comunistas "estalinistas"), integradas por ritos masivos y permanentes, capaces de homogeneizar las propiedades sociales de su población, han aportado tanta, si no más creatividad intelectual, que la bohemia.

Así, si bien la tendencia de Bourdieu muestra su valor heurístico en otros campos, debería confrontarse, como él mismo reclama (HA 149) con estudios más complejos y variados sobre la relación entre propiedades sociales, posición académica y producción cultural. Lo que requeriría, se insiste de nuevo, otro tipo de elección de la población estudiada (recortada en Homo academicus en torno a la consagración institucional y al reconocimiento de los pares) y, por supuesto, estudios a la vez más profundos y más amplios de cómo se conecta la vida cultural con los recursos sociales de los individuos.

Pese a su separación, entre ambos poderes, el científico y el universitario, funciona una complicidad de fondo. En primer lugar porque estructuran el campo de lo posible, a partir de la justificación que le proporcionan a cada uno las debilidades del otro. El poder universitario se refuerza en la búsqueda compulsiva de la innovación, a menudo intelectualmente inane, que caracteriza a muchas vanguardias culturales y científicas. Los productores culturales, por su parte, se justifican en todo cuanto no sea académico, y tienen su contramodelo en una vida universitaria cortesana y una producción intelectual reiterativa.

Más allá de ambos poderes, se encuentra la creación científica y cultural. El reconocimiento intelectual no supone inmediatamente la calidad creativa de un trabajo. Ésta se mide por su capacidad para interesar cuando nos alejamos del espacio cultural y del segmento temporal en que ha sido producido. La reputación intelectual se mide en el corto plazo, la creatividad exige conectarse con problemas definidos por un campo intelectual de historia larga. A menudo, los prestigios mundanos ensalzan lo que no es sino ofrenda al aire del tiempo. Sólo con estas salvedades, se asume el valor heurístico de la distinción formulada por Bourdieu (1998: 236), de gran potencia para la sociología de los intelectuales, entre producción de ciclo corto (que responde a las expectativas establecidas... y que fenece con ellas) y producción de ciclo largo que subsiste a su momento de surgimiento y que sigue produciendo efectos incluso cuando los marcos culturales en los que se gestó desa- 
parecen. El reconocimiento de los pares tiene su posibilidad brillante (ser reconocido por los intelectualmente consagrados) pero también su posible funesto (convertirse en mero reproductor de una ideología con signos científicos que responde a una demanda no científica). Algo que sucede, muestra Bourdieu, cada vez más y debido a la influencia creciente de la consagración periodística en la vida intelectual. Las apariencias científicas permiten conquistar un público constituido por estudiantes y profesores, pendiente de las modas culturales y, dada su escasa especialización, incapaz de comprender los matices y las exigencias del trabajo científico. Para satisfacerlos, los investigadores asumen el estilo ensayístico y comparten con el periodismo la repulsa de la pedantería académica. La conquista de un público para tales productos exige cada vez más la complicidad del periodismo cultural y el mundo editorial. El diálogo con la tradición intelectual y con la vanguardia científica, condiciones de un verdadero trabajo creativo en ciencias humanas, puede ser contraproducente para conectar, en esas condiciones, con audiencias amplias.

Por otra parte, la colonización de la vida científica, no procede solo del mercado de la cultura, sino también del mundo de la administración. La investigación por encargo exige, por una parte, el desarrollo de competencias para la captación de fondos públicos y privados y la gestión empresarial de grupos de investigación y, por otra parte, la adaptación a los expertos que evalúan los resultados, las líneas de trabajo y las metodologías.

En fin, el campo universitario es el lugar de competencia de poderes plurales. Debido a que los campos necesitan tratar con su exterior, la presión de poderes externos al mismo redefine sus fronteras: las transformaciones demográficas exigen retener o animar el orden sucesorio, la industria cultural impone modelos científicos, la gestión administrativa de la investigación prácticas industriales de producción intelectual.

\section{La explicación sociológica del acontecimiento}

Tras el mapa, la narración. Aunque se reclame de valores universales o se identifique con causas ajenas a su medio, un universitario actúa siempre según la lógica de su propio campo y sus opciones son siempre, indefectiblemente, signos que dirige a sus colegas y contendientes. Mayo del 68 fue el resultado de transformaciones del mundo universitario, fundamentalmente de la crisis que produjo, durante los años 1960, la elevación del número de estudiantes universitarios y, por consiguiente, del profesorado. Ese aumento no generó idénticos efectos en todas las disciplinas, por la sencilla razón que no existe un espacio universitario unificado y cada mercado local tiene sus jerarquías y sus valores propios (HA, 180). Los efectos, por consiguiente, exigen una descripción circunstanciada, algo que muestra lo que ya se 
señaló antes: la hipótesis de Bourdieu acerca del mundo social no destruye como un buldózer las peculiaridades del mismo, pese a que éstas contengan complicaciones -la existencia de jerarquías diversas a las que se perciben cuando se mira el campo social desde la cúspide- respecto a las versiones más esquemáticas de la visión bourdisiana del espacio social.

Las disciplinas menos instaladas académicamente disponían de menos reserva de personal adaptado al orden institucional. Los ritmos de sucesión dejaron de funcionar según lo previsto y dos tipos de actores entraron en juego. En primer lugar, individuos que habían seguido la vía ortodoxa de acceso y que descubrieron que su promoción ya no les daba lo que ellos esperaban: muchos vieron sus carreras estancadas y no accedieron a los puestos ansiados. En segundo lugar, aquellos que, sobre todo en las disciplinas menos académicas, acceden sin el entrenamiento correspondiente y aceptaban mal las imposiciones del orden establecido. Las complicidades se rompen, por tanto, desde dos criterios diferentes e incompatibles: la defensa de los antiguos privilegios y aquellos que contestan las fases y los ritmos que pautaban el acceso a los mismos. Ninguno de tales grupos proponía el programa que hubiera deseado Bourdieu: "Un orden en el que el reclutamiento y el avance profesional dependería únicamente de los criterios de productividad y de eficacias pedagógicas y científicas" (HA, 205).

La crisis del sistema de enseñanza afectó a la sociedad en su conjunto. En primer lugar, por el papel cada vez más importante que juega la enseñanza en la reproducción del sistema de clases. En segundo lugar, por la distancia entre las esperanzas -alimentadas por un sistema anterior de exigencias y retribuciones- de los universitarios y las recompensas efectivas, menores de las esperadas, y que generaron un ambiente de revuelta generalizado. La frustración fue mayor entre los procedentes de los grupos dominantes, si bien los efectos de la pérdida de valor de los títulos fueron mayores entre los alumnos de clase trabajadora. Estos, sin embargo, no juzgan el mundo de la misma manera que la clase dominante. En primer lugar, porque tienen más tolerancia a la frustración y, en segundo lugar, porque los mercados locales de las clases populares, que no funcionan todos con la perspectiva de la Rue d'Ulm, siguen valorando lo que el análisis estadístico puede considerar una devaluación objetiva (HA, 214). En cualquier caso, la crisis de los vástagos de la burguesía encontró ecos entre fracciones de las clases medias con sentimientos similares e incluso entre jóvenes obreros o campesinos frustrados por los escasos frutos de la enseñanza primaria o secundaria. La crisis de los títulos, pese a que tuvo efectos diferentes según las clases, unificó poblaciones heterogéneas. ${ }^{5}$

\footnotetext{
5 Louis Gruel (2004) ha criticado el modelo explicativo de Bourdieu insistiendo en, primero, la inexistencia de devaluación de los títulos, segundo, que la rebelión no se comprende bien desde el mundo universitario y, tercero, que se trato de una crisis de transmisión intergeneracional de un universo cultural. La discusión del importante trabajo de Gruel desborda el espacio de este artículo.
} 
En las facultades de letras confluyeron múltiples dinámicas: en primer lugar, sobre todo en las disciplinas nuevas, se refugiaron jóvenes burgueses de rendimiento escolar escaso y con grandes aspiraciones, en segundo lugar, estudiantes de clases medias que no habían podido conseguir otros estudios y con poco capital social que les permita rentabilizar los títulos, en tercer lugar, profesores incorporados rápidamente sin el entrenamiento preceptivo de sumisión universitaria. Agentes procedentes de campos diferentes se encontraban en posiciones homólogas, aunque entre el hijo de un maestro que no encuentra empleo como sociólogo y el de un intelectual que accede a la docencia universitaria en condiciones precarias, las distancias son enormes. Esas identificaciones engañosas sólo existen durante la crisis, después, cuando el orden social se reimpone, cada uno persigue sus objetivos respectivos, en muchos casos, enfrentados. Las crisis producen comunidades emocionalmente densas capaces de agrupar gentes que de ordinario estarían separadas (HA, 229), algo que la teoría de la acción racional es incapaz de comprender.

La comunicación entre poblaciones alejadas que caracteriza las crisis permite a Bourdieu una rica fenomenología sociológica de la experiencia revolucionaria. En primer lugar, se sincronizan existencias, las de los dominantes y las de los dominados, que juegan en mundos diferentes. Dado que los dominantes juegan en más campos que los dominados, estos tienden a ser más sinceros, más profundamente juramentados con sus opciones vitales. Los de arriba, sin embargo, tienen siempre que guardar fidelidades más complejas, lo que les exige "vidas paralelas". Cuando el fin de la crisis revele esa multiplicidad de referencias, la sensación de traición embargará a los dominados. En segundo lugar, y relacionado con lo anterior, la representación de los actores disuelve la complejidad social y la resume en dos bandos: ellos y nosotros, los dominantes y los dominados, esquema de base de todos los jdanovismos. La crisis economiza brutalmente las diferenciaciones simbólicas y las retraduce en términos políticos. La dinámica interna de los universos sociales, que puede separar radicalmente a dos individuos, queda abolida situando juntos a agentes heterogéneos socialmente y separando a otros mucho más próximos. En tercer lugar, lejos de ser una simple engañifa, la situación revolucionaria disuelve la relación habitual con el mundo: ni el pasado parece pesar (disuelto en la interacción ritual eufórica) ni el futuro impone una conducta de cálculo en el presente. Por tanto, la experiencia revolucionaria no atrae a todos por igual. Los más asentados la contemplan como el Apocalipsis y la recaída en la Barbarie mientras los menos integrados, por más jóvenes o por menos beneficiados por el sistema, tienden a simpatizar con las oportunidades que abre. En cuarto lugar, las situaciones de eclosión revolucionaria favorecen a aquellos que se encuentran entrenados en la gestión de acontecimientos políticos, y saben condensar semánticamente posiciones muy dispersas. En el fondo, pese a la reivindicación de la espontaneidad, Mayo favoreció a quienes se encontraban entrenados en los aparatos políticos de ultraizquierda, que 
hasta entonces carecían de audiencia. Bourdieu, como muchos analistas, encuentra en el estilo faltón y agresivo de los líderes de Mayo parte de la cultura de las clases dominantes "encanalladas" que según Perry Anderson (1998: 86) controlan el capitalismo actual.

\section{Conclusión}

La lectura del trabajo de Bourdieu nos revela su enorme poder de inspiración. Siempre con ciertas reservas: en el nivel epistemológico, siempre que reconozca la pluralidad legítima de acercamientos científicos, algo que se deriva lógicamente de su diferencia entre individuo empírico y epistémico; en el nivel estructural, siempre que la teoría de la homología entre los universos sociales sirva de herramienta heurística y no de mecánica metafísica que ahoga el trabajo empírico. Por ejemplo, creyendo que el mundo de la cúspide intelectual parisina sirve para conocer la realidad universitaria, intelectual o científica.

En el nivel histórico, Bourdieu explica la crisis como la conjunción coyuntural de dinámicas diversas, capaces de producir novedades con distinto nivel de persistencia. Como modelo es intachable. Pero, claro está, cabe objetarle si el mundo universitario, tal y como lo estudió (en las alturas de los catedráticos), sirve para comprender los acontecimientos de Mayo.

En cualquier caso, hay que leer y releer Homo academicus, incluso si se cuestiona el modelo teórico de Bourdieu, si se discute sobre cómo lo vuelve operativo $\mathrm{o}$, incluso, si se consideran sus conclusiones demasiado arriesgadas. Con todas esas reservas, el libro nos recuerda cuáles son las decisiones filosóficas previas de una investigación, cómo éstas permiten elaborar hipótesis a partir del funcionamiento del mundo social y, a partir de éstas, confrontarse con la realidad empírica y obtener resultados que no sospechábamos. Por abierto a la crítica que se encuentre el libro, es difícil no sorprenderse de lo alto que deja el listón científico para quienes estudien el campo intelectual.

\section{Referencias bibliográficas}

Anderson, P. (1998): The Origins of Postmodernity, London, Verso.

Baranger, D. (2009): "Para el estudio de los campos universitarios: Pierre Bourdieu y la construcción del objeto en Homo Academicus", Pensamiento Universitario, $\mathrm{n}^{\mathrm{O}} 12$.

Bourdieu, P. (1980): Le sens pratique, Minuit, Paris.

Bourdieu, P. (1984) Homo academicus, Minuit, Paris. 
Bourdieu, P. (1998): Les règles de l'art. Genèse et structure du champ littéraire, Paris, Seuil.

Bourdieu, P. (2001): Science de la science et réflexivité, Paris, Raisons d'agir.

Bourdieu, P. (2004): Esquisse pour une auto-analyse, Paris, Raisons d'agir.

Delsault, Y. (2002): «Pierre Bourdieu et Yvette Delsault entretien. Sur l'esprit de la recherche», Y. Delsault, M.-C.- Rivière, Bibliographie des travaux de Pierre Bourdieu sui d'un entretien sur l'esprit de la recherche, Pantin, Le Temps des Cerises.

Collins, R. (2000): The sociology of philosophies. A global theory of intellectual change, Cambridge, Massachussetts, y Londres, Harvard University Press.

Foucault, M. (2001): L’herméneutique du sujet. Cours au Collègue de France. 1981-1982, París, Gallimard-Seuil.

GRUEL, L. (2004): La rébellion de 68. Une relecture sociologique, Rennes, PUR. Moreno Pestaña, J. L. (2009) «Pour une sociologie de l'échec intellectuel», EspacesTemps.net, Textuel, http://espacestemps.net/document7864.html

PASSERON, J.-C. (2006): Le raisonnement sociologique. Un espace non poppérien de l'argumentation. Paris, Albin Michel.

José Luis Moreno Pestaña

Universidad de Cádiz

joseluis.moreno@uca.es 